\title{
CLASSIFICAÇÃO DE TRÁFEGO COM TÉCNICAS DE DEEP LEARNING PARA GERÊNCIA DA SEGURANÇA NO CONTROLE DE REDES SDN
}

\author{
Ludimila O. Félix, Mateus Romani, Georges Daniel Amvame-Nze, \\ Rafael Timóteo de Sousa Júnior, Robson de Oliveira Albuquerque \\ e Bruce William Percílio Azevedo \\ Programa de Pós-Graduação Profissional em Engenharia Elétrica (PPEE), Departamento de Engenharia Elétrica, \\ Universidade de Brasília, Brasília, Brasil, Zipcode 70910-900
}

\begin{abstract}
RESUMO
A combinação do modelo de Software Defined Networks (SDN) com algoritmos de aprendizado de máquina permite vislumbrar a possibilidade de redes definidas por aprendizado, ou seja, redes que utilizam conhecimentos dos fluxos de tráfego para gerenciar sua configuração e funcionamento. Nesse contexto, as possibilidades que emergem são infinitas, incluindo a previsão de tendências na rede (por exemplo, prevendo possíveis congestionamentos) e engenharia de tráfego. Essas funções não só contribuem para a disponibilidade da rede, mas também constituem pilares da gerência de segurança de uma rede por empregarem identificação e classificação de tráfego nos aspectos de controle de acesso, filtragem por firewalls adaptáveis e detecção de ataques DoS e DDoS. No presente artigo, é apresentada uma proposta de classificação de tráfego para uso na segurança em redes SDN utilizando aprendizado profundo, Deep Learning por Multi-Layer Perceptron (MLP).
\end{abstract}

\section{PALAVRAS-CHAVE}

Software Defined Networks, Classificação de Tráfego, Machine Learning

\section{INTRODUÇÃO}

As redes definidas por software, do inglês Software Defined Network (SDN) introduz um conceito de rede com arquitetura logicamente centralizada, que separa o plano de controle do plano de dados. Esse paradigma apresenta uma solução para o aperfeiçoamento do uso dos recursos de uma rede, através de sua visão global. As novas abordagens da internet do futuro estão trazendo novos desafios de acessibilidade, largura de banda e gestão dinâmica dos recursos [Wenfeng 2015].

Nas redes convencionais, para cada dispositivo de rede (componentes de hardware), protocolos e conjuntos de regras são implementados funções de controle e monitoramento do fluxo de dados, gerenciamento do roteamento e interconexão dos dispositivos da rede [Fei 2015]. Há diversas possibilidades que surgem da aplicação de aprendizado de máquina no contexto de redes de comunicação, como por exemplo, a previsão de volume de tráfico, monitoramento de segurança: controle de acesso, firewall adaptativo, detecção de DoS e DDoS, e previsão de congestionamento [Amaral 2016]. Uma das vantagens trazidas pelas redes definidas por software é a facilidade de implementação e gerenciamento. A separação da camada de dados e a camada de controle, e ainda, a visualização centralizada da rede proporciona uma arquitetura dinâmica, gerenciável, adaptável [Joëlle 2018].

O surgimento do SDN trouxe novas perspectivas para a realização da classificação de tráfego e seleção de recursos. Devido ao mecanismo do protocolo OpenFlow é possível personalizar para reunir recursos de fluxo e indicar política de encaminhamento para cada switch. Com o rápido desenvolvimento da SDN, o número de trabalhos relacionados ao estudo de classificação de tráfego em SDN também emergiu. O número de trabalhos que classificam o tráfego utilizando algoritmos clássicos de aprendizado de máquina é mais extenso, como árvores de decisões e Máquinas de Vetor e Suporte (SVM). 
O intuito desse trabalho é propor um método de classificação de tráfego baseado em redes neurais artificiais Multi-Layer Perceptron (MLP). O trabalho apresentará inicialmente conceitos importantes para a trabalho, em seguidas, os resultados iniciais da pesquisa.

\section{CONTROLE DE REDES}

A tabela 1 apresenta o resumo das três formas de classificação de tráfego encontradas na literatura.

Tabela 1. Resumo da classificação de tráfego

\begin{tabular}{|c|c|}
\hline Classificação & Descrição \\
\hline $\begin{array}{l}\text { Tráfego IP } \\
\text { Baseada em } \\
\text { portas }\end{array}$ & $\begin{array}{l}\text { Uma forma de classificação do tráfego é pela porta de encaminhamento, afinal, o número de portas } \\
\text { é fixado pelo Internet Assigned Numbers Authority (IAN). Essa forma de classificação não é mais } \\
\text { viável, afinal, o uso de proxy, a existência de protocolos e aplicações que escondem ou usam números } \\
\text { randômicos ou dinâmicos das portas relacionada com cada tráfego torna impossível a associação do } \\
\text { tráfego com a porta correta. Além disso, alguns trabalhos, mostram a ineficiência não é eficiente, } \\
\text { tendo entre } 30 \% \text { e } 70 \% \text { de erro na identificação do fluxo do tráfego [Jinghua 2018]. }\end{array}$ \\
\hline
\end{tabular}

Os sistemas de análise de payload, também conhecidos como Deep Packet Inspection (DPI), usam expressões regulares e identificação de padrões em um fluxo de pacotes, no sentido de tomar decisões Tráfego IP sobre a rede. Isso permite a classificação do tráfego e o controle da rede com base nos fluxos de Baseado no tráfego, conteúdo e aplicação [India 2014]. Tais sistemas apresentam problemas de escalabilidade à Payload medida em que se aprofunda o nível de análise. Várias técnicas vêm sendo utilizadas para reduzir o problema de escalabilidade, incluindo as técnicas de Fast Finite Automata, Deterministic Finite Automata, além de soluções de hardware como Graphic Processor Units e Single Instruction Multiple Threads.

A ideia das propostas é aproveitar do comportamento estatístico do fluxo de tráfego de rede (como o comprimento de pacotes, tempo de chegada de pacotes e assim por diante) que são únicos para um tipo específico de protocolo ou aplicação [Xue 2013]. Existem três tipos de algoritmos de aprendizado de máquina: Aprendizado supervisionado, não supervisionado e semi-supervisionado.

Tráfego IP usando $\mathrm{O}$ aprendizado de máquina supervisionado é capaz de classificar fluxos de pacotes com algoritmos

Machine como, Support Vector Machines, Árvore de Decisão, Regressão Logística, entre outros. Para isso é necessário obter dados rotulados, algo desafiador de se obter em um fluxo de rede. O uso de algoritmos não supervisionados, p ode superar a dificuldade de se obter dados rotulados. Essa técnica geralmente é usada para construir agrupamentos ou, clusters, de acordo com as similaridades que os dados apresentam. Há diversos algoritmos que podem ser usados com aprendizado não supervisionado como K-means e Self Organizing Maps [Amaral 2016]. O aprendizado profundo ou deep learning, é uma técnica de aprendizado de máquina que se vale da capacidade de criação de camadas de neurônios, o que caracteriza os algoritmos de redes neurais artificiais.

\section{RESULTADOS}

Neste trabalho foi utilizado amostras de um fluxo de tráfego rotulado unidimensional entre um host e um switch de borda: o endereço IP do emissor, endereço IP do destino, número da porta de encaminhamento e número da porta de destino, protocolos da camada de transporte, tempo de início, tempo de fim, duração e tamanho dos primeiros 10 pacotes.

Para melhor eficiência do classificador foi necessária uma filtragem da base de dados das amostras do fluxo de tráfego: ignoramos instâncias repetidas e outliers. A base foi dividida em dois grupos: $80 \%$ para treinamento e $20 \%$ para testar essa rede. Além disso, foi rotulado o tráfego coletado. A Tabela 2 apresenta alguns dos rótulos do classificador proposto nesse artigo.

Os rótulos são baseados no número das portas de encaminhamento e suas aplicações e categorias. Para construção dessa lista, foi utilizado as informações do IANA. Foi realizado 10 ciclos de aprendizados de uma rede MLP, onde consideramos as 7 saídas possíveis, conforme Tabela 2 de rotulação dos tráfegos. Para realizar o treinamento e extrair as informações desse classificador, usufruímos da toolbox do Matlab. O número de 
neurônios na camada oculta foi validado de forma a atingir um ponto ótimo entre precisão e custo computacional, no caso, concluiu-se que sete neurônios na camada oculta teve o melhor desempenho. Demonstrando a alta complexidade do nosso classificador.

Tabela 2. Rótulos para categorização do classificação de tráfego

\begin{tabular}{ll}
\hline Categoria & Aplicação e Protocolos \\
\hline Web & HTTP, HTTPS \\
P2P & BitTorrent, FastTrack, HotLine, Ares \\
Mail/News & BIFF, SMTP, POP, IMAP, IDENTD, NNTP \\
Streaming & MMS(WMP), Real, Quicktime, Shoutcast, Logitech Video IM, Backbone Radio \\
Encryption & SSH, SSL, Kerberos, IPSec, ISAKM \\
Games & Minecraft, WOW, LOL, Age of Empires \\
Chat & MSN Messenger, Yahoo messenger, ICQ \\
\hline
\end{tabular}

Outra métrica interessante para avaliar a qualidade da nossa classificação, são acurácia, taxa de falsos positivos (TP), taxa de positivos verdadeiros (sensibilidade). Para os 10 ciclos, tivemos 78,8799\% como sendo a melhor acurácia do classificador, a sensibilidade para esse caso foi 0,79 e 0,2111 de falsos positivos.

\section{CONCLUSÃO}

O classificador proposto nesse trabalho para classificação de tráfego e consequentemente o controle da rede mostrou-se promissor para o problema proposto. A ideia é expandir esse trabalho para uma classificação on-line. E testar a solução para a classificação de larga escala de tráfego. E consequentemente expandir para resolver problemas de ataques em redes SDN.

\section{AGRADECIMENTO}

Os autores agradecem o apoio das Agências brasileiras de pesquisa, desenvolvimento e inovação CNPq (Projeto INCT SegCiber 465741/2014-2), CAPES (Projetos FORTE 23038.007604/2014-69 e PROBRAL 88887.144009/2017-00) e FAPDF (Projetos UIoT 0193.001366/2016 e SSDDC 0193. 001365/2016), bem como o suporte do Laboratório LATITUDE/UnB (Projeto SDN 23106. 099441/2016-43), e as cooperações com o Ministério da Economia (TEDs DIPLA 005/2016 e ENAP 083/2016) e o Gabinete de Segurança Institucional da Presidência da República (TED 002/2017).

\section{REFERÊNCIAS}

Amaral Pedro et al., 2016, Machine Learning in Software Defined Networks: Data collection and traffic classification in IEEE 24th International Conference on Network Protocols (ICNP).

Fei Hu et al., 2014, A Survey on Software-Defined Network (SDN) and OpenFlow: From Concept to Implementation in IEEE Communications Surveys and Tutorials, Vol. 16, No. 4, pp.2181-2206.

India, 2014, Deep Packet Inspection, Department of Telecomunications - Government of India.

Jinghua Y., Jing Y., 2018, A Survey of Traffic Classification in Software Defined Networks, 1st IEEE International Conference on Hot Information-Centric Networking.

Joëlle M. M. et al., 2018, Strategies for detecting and mitigating DDoS attacks in SDN: A survey in Journal of Intelligent \& Fuzzy Systems, Vol. 13 No. 1.

Wenfeng Xia et al., 2015, "A Survey on Software-Defined Networking", IEEE Communications Surveys and Tutorials, Vol.17, No.1,pp. 27-51.

Xue et al., 2013, Traffic Classification: Issues and Challenges in Journal of Communications Vol. 8.4, pp. 240- 248. 